INRA Prod. Anim., 1996, 9 (1), 45-60

\author{
P. CHEMINEAU, B. MALPAUX, \\ J. PELLETIER, B. LEBOEUF*, \\ J.A. DELGADILLO **, \\ F. DELETANG ***, T. POBEL ***, \\ G. BRICE ****
}

INRA Neuroendocrinologie Sexuelle, Physiologie de la Reproduction 37380 Nouzilly

* INRA Station Expérimentale d'Insémination Artificielle 86480 Rouillé

** Univ. Autónoma Agraria A. Narro, Torreon, Coah., Mexique

*** SANOFI Santé Nutrition Animale 33501 Libourne Cedex

**** Institut de l'Elevage, Chemin de Borde Rouge, 31321 Castanet Tolosan

\section{Emploi des implants de mélatonine et des traitements photopériodiques pour maîtriser la reproduction saisonnière chez les ovins et les caprins}

\title{
Résumé
}

La mélatonine est une substance naturellement sécrétée par la glande pinéale pendant la période obscure du nycthémère, qui transmet l'information photopériodique chez les mammifères. L'administration continue par un implant sous-cutané, comme dans les expérimentations décrites ici, permet de mimer les jours courts alors que les yeux des animaux perçoivent les jours longs naturels du printemps et de l'été. Il est nécessaire de faire subir une alternance de jours longs et de jours courts pour pouvoir maîtriser la période d'activité sexuelle.

Chez la brebis conduite en lutte naturelle, un implant sous-cutané de mélatonine (Mélovine®) est inséré de 30 à 40 jours avant l'introduction des béliers. Les différents essais réalisés depuis plusieurs années chez 5 races françaises, et qui mettaient en comparaison, dans les mêmes élevages, des femelles traitées et des femelles témoins, montrent que la fécondité des brebis traitées est très supérieure à celle des brebis témoins (16 agneaux nés en plus pour 100 brebis mises en lutte). Les dates moyennes de mise bas sont plus précoces et moins étalées chez les traitées que chez les témoins. Chez la brebis également, cette fois-ci en association avec un traitement hormonal de synchronisation de l'œstrus et une insémination artificielle, la fécondité des brebis traitées, pour l'ensemble ostrus induit plus retours, est aussi très significativement supérieure à celle des brebis témoins (30 agneaux nés en plus pour 100 brebis mises à la reproduction).

Chez la chèvre, du fait de la forte demande existante pour une lutte en pleine contre-saison (avril à juillet), il est recommandé de faire subir un traitement lumineux (éclairement supplémentaire avec aube fixe et " flash " nocturne) pendant une période d'au moins 2 mois avant la pose de l'implant de mélatonine. Les boucs recoivent le même traitement ; les femelles sont séparées de tout contact avec les mâles à partir de la pose de l'implant. La lutte naturelle se fait en introduisant les boucs traités parmi les femelles, de 35 à 70 jours après la pose de l'implant, de façon à bénéficier de «l'effet bouc ». Dans ces conditions, la fertilité est voisine de celle observée en lutte naturelle pendant la saison sexuelle (supérieure à $80 \%$ ) et les fécondations ont lieu environ 10 jours après l'introduction des mâles. La prolificité est équivalente à celle observée en saison sexuelle. L'association de ce traitement avec un traitement progestatif classique de synchronisation hormonale des œstrus et avec l'insémination artificielle est en cours d'essai.

Chez le bélier, l'insertion d'implants permet une avance de la croissance testiculaire et une amélioration de la production spermatique. Si l'on souhaite obtenir une activité spermatogénétique intense en pleine contre-saison, comme chez les mâles des centres d'IA, il est nécessaire de faire précéder la pose de l'implant d'une période de jours longs réels ou mimés par une heure d'éclairement nocturne. Un tel traitement stimule la croissance testiculaire, améliore le comportement sexuel, la production spermatique et augmente la fertilité après IA, par rapport aux béliers non traités. Il est également possible de faire subir aux béliers reproducteurs une alternance d'un mois de jours longs et d'un mois de jours courts, ce qui induit le maintien d'une activité spermatogénétique élevée et constante pendant plusieurs années. En bâtiment ouvert, sur des mâles laissés en photopériode naturelle, l'alternance d'un mois d'éclairement nocturne avec un mois d'implant de mélatonine permet d'aboutir aux mêmes résultats, la dépose de l'implant en fin de traitement devenant toutefois délicate.

Chez le bouc, le même type d'alternance rapide entre jours longs et jours courts permet de maintenir une production spermatique élevée de bonne qualité pendant au moins trois années consécutives. Dans ces conditions, le nombre de doses de semence congelée produites est très supérieur chez les animaux traités par rapport aux animaux témoins $(+41$ à $+69 \%)$. La fertilité des chèvres inséminées artificiellement avec de telles doses n'est pas différente de celle des chèvres inséminées avec la semence des boucs témoins.

Les implants de mélatonine Mélovine®, ont obtenu en 1995 l'autorisation de mise sur le marché. 
Les ovins et les caprins originaires des zones tempérées manifestent d'importantes variations saisonnières de leur activité sexuelle. Chez les deux sexes, il existe une période d'activité sexuelle maximum qui s'étend, en général, d'août à janvier et une période d'activité minimum de février à juillet. Les variations se manifestent, chez la femelle, par l'existence d'une période d'anœstrus saisonnier et, chez le mâle, par une diminution de l'intensité du comportement sexuel, de la production spermatique en quantité et en qualité, entraînant des baisses plus ou moins importantes de fertilité et de prolificité dans les troupeaux (Thimonier 1989).

Ces variations sont sous la dépendance des changements dans la durée de l'éclairement quotidien (photopériode). Les jours courts sont stimulateurs de l'activité sexuelle et les jours longs inhibiteurs de celle-ci, chez les petits ruminants. Il n'existe cependant aucune durée du jour constante permettant le maintien d'une activité sexuelle permanente. Il s'établit des états réfractaires soit aux jours longs, soit aux jours courts. La maîtrise de l'activité sexuelle n'est possible que par une alternance de jours longs et de jours courts, alternance qui existe dans les conditions naturelles. La mélatonine, substance naturelle synthétisée dans la glande pinéale, est le messager biochimique qui permet au système neurœendocrinien des animaux de mesurer la durée de l'éclairement quotidien. Cette mélatonine n'est, en effet, sécrétée que pendant la phase obscure et c'est grâce à la durée de cette sécrétion que les animaux perçoivent la durée de la nuit et donc du jour (Karsch et al 1984). Des jours courts peuvent être mimés par une libération constante de mélatonine grâce à des implants sous-cutanés, à condition que ce traitement soit appliqué après qu'une période "suffisante " de jours longs ait " levé " l'état réfractaire aux jours courts.

\section{1 / La mélatonine : une substance naturelle utilisable pour la maîtrise de la reproduction}

La mélatonine (figure 1) est une substance naturellement présente dans l'organisme de tous les mammifères et presque tous les vertébrés. Elle est synthétisée, principalement dans la glande pinéale, à partir du tryptophane et de la sérotonine, sous l'effet d'enzymes dont l'activité est commandée par la perception jour/nuit (Collin et al 1988). Synthétisée et sécrétée uniquement pendant la période nocturne, elle présente des concentrations dans le sang périphérique multipliées au moins par 50 à l'occasion du passage lumière/obscurité (Ravault et Thimonier 1988 ; figure 2).

Cette sécrétion élevée se maintient pendant toute la période obscure. Elle s'arrête le jour suivant lorsque la lumière stimule à nou-
Figure 1. Structure de la mélatonine (poids moléculaire 232).<smiles>COc1ccc2[nH]ccc2c1</smiles>

Figure 2. Concentration plasmatique moyenne de mélatonine chez des brebis lle-de-France, maintenues en jours courts et en jours longs, avec et sans implant de mélatonine (d'après Chemineau et al 1993).

La zone noire représente la période obscure du nycthémère.

Mélatonine (pg/ml plasma)
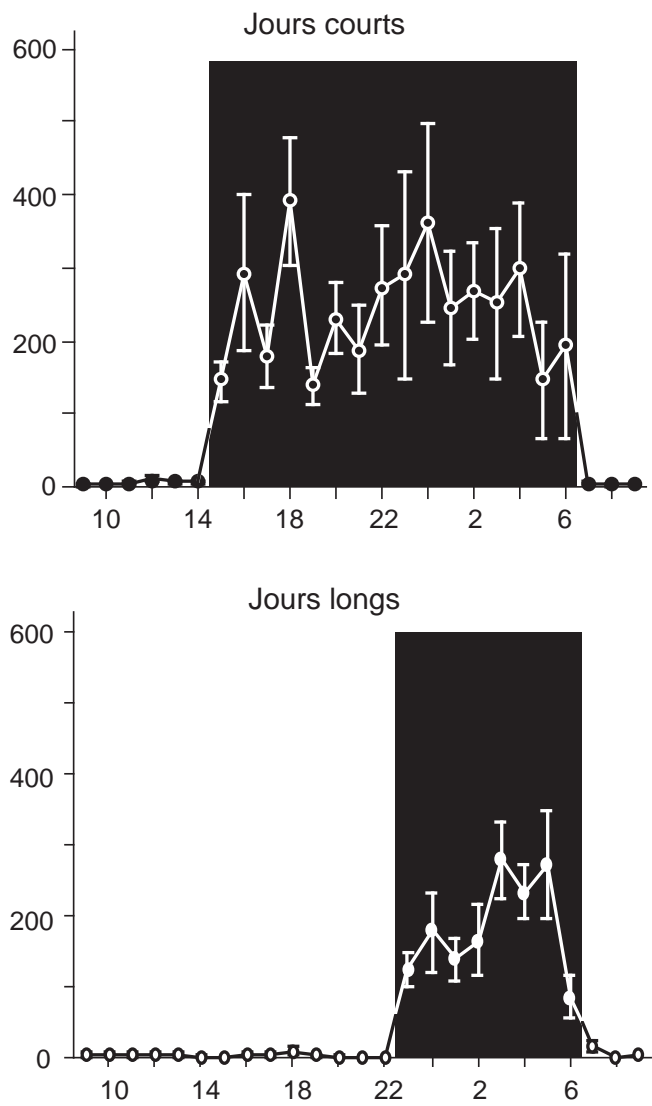

Jours longs + implant

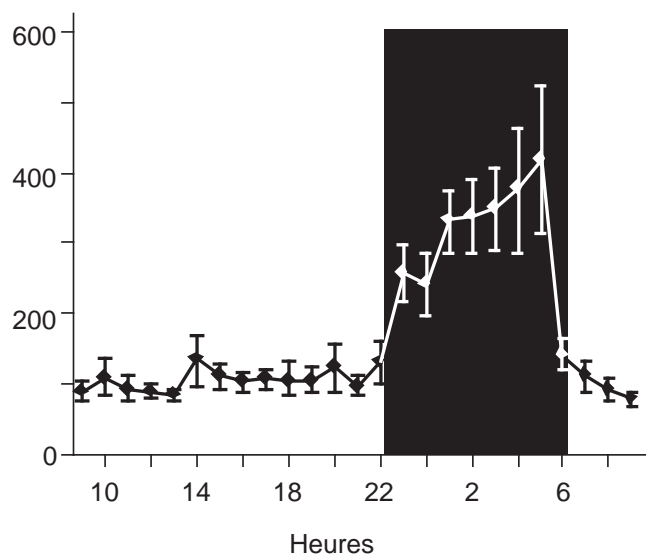


veau la rétine, puis les noyaux suprachiasmatiques et enfin la glande pinéale.

C'est grâce à la durée de cette sécrétion que les brebis, comme tous les mammifères étudiés, sont capables de mesurer la durée de la nuit, et donc celle du jour (Bittman et al 1983, Ravault et Thimonier 1988). Dans les races de brebis qui présentent des variations saisonnières marquées d'activité sexuelle, races ovines d'Europe du Nord notamment (Pelletier et al 1987), la mélatonine, par l’intermédiaire de sa durée de sécrétion contrôle ces variations d'activité sexuelle au cours des saisons. Lorsque la durée de sécrétion de mélatonine est longue, les brebis interprètent cette durée comme un jour court et déclenchent leur activité sexuelle (Bittman et al 1983), et ceci même si leurs yeux perçoivent des jours longs.

Si l'action de la mélatonine sur la reproduction saisonnière des brebis est désormais bien connue, son mode d'action sur le système nerveux central reste encore imprécis. Récemment, des sites récepteurs ont été identifiés. Deux zones bien distinctes sont considérées comme des cibles potentielles de la mélatonine quant à son effet sur la fonction de reproduction :

- la pars tuberalis de l'hypophyse (zone entourant l'éminence médiane) possède de nombreux récepteurs à la mélatonine (de Reviers et al 1989), mais l'insertion de microimplants de mélatonine à son contact ne provoque aucune stimulation de la fonction de reproduction (Malpaux et al 1994),

- l'hypothalamus médiobasal est le seul site hypothalamique où l'insertion de microimplants de mélatonine cause une stimulation de la fonction de reproduction (Malpaux et al $1993 \mathrm{~b})$. Une très faible densité de récepteurs est, pour le moment, identifiée dans ce site.

\section{1 / Activité de la mélatonine}

La mélatonine est intéressante pour la reproduction en avance de saison (1,5 mois maximum par rapport à la date normale de reproduction sans traitement) chez les petits ruminants. Cette activité a été clairement démontrée sur de nombreuses races de brebis dans le monde. Ainsi, des brebis croisées Suffolk, traitées à partir du 18 juin par de la mélatonine sous forme d'implants sous-cutanés ou sous forme de distribution quotidienne dans l'alimentation, démarrent leur saison de reproduction respectivement 52 et 50 jours plus tôt que les brebis témoins non traitées. Ce démarrage plus précoce est identique à celui de brebis placées en jours courts, également à partir du 18 juin, qui, elles, manifestent une avance de 42 jours par rapport aux témoins (English et al 1986).

Beaucoup d'autres auteurs ont rapporté une telle efficacité pour le déclenchement précoce de l'activité sexuelle chez la brebis et notamment Kennaway et al (1982), Nett et Niswender (1982), Arendt et al (1983), Nowak et Rodway (1985), Poulton et al (1987), Mori et al (1987), Stellflug et al (1988), Ronayne et al (1989), Staples et al (1991).

\section{2 / Importance du mode de distribution de la mélatonine}

La régularité et la durée du traitement par la mélatonine sont des impératifs importants. L'application quotidienne d'un traitement mélatonine est indispensable au déclenchement précoce de l'activité. Ainsi, des brebis Suffolk et Cheviot recevant de la mélatonine une fois ou trois fois par semaine seulement, déclenchent leur activité ovulatoire à la même date que les animaux témoins. En revanche, les femelles recevant celle-ci quotidiennement ou portant un implant souscutané permettant une libération constante, déclenchent leur activité un mois plus tôt que les témoins (Ronayne et al 1989).

Une administration quotidienne ou une libération permanente est donc indispensable au déclenchement précoce de l'activité sexuelle de la brebis.

De la même façon, des trop faibles durées d'administration de mélatonine par voie souscutanée ne permettent pas d'obtenir une réponse satisfaisante. La durée optimale, pour obtenir un déclenchement plus précoce des ovulations chez au moins les $2 / 3$ des animaux traités et une cyclicité ovarienne régulière, est supérieure à 36 jours mais inférieure à 93 jours (Nowak et Rodway 1987). La durée optimale pour un traitement sous forme d'implants sous-cutanés est sans doute située aux alentours de 70 jours.

La dose de mélatonine libérée de manière régulière doit permettre d'obtenir des concentrations plasmatiques voisines de $50 \%$ du niveau moyen nocturne des animaux témoins, pour aboutir à une avance de l'activité ovulatoire. En-dessous de ce seuil, la réponse des brebis semble dépendre de leur niveau endogène de mélatonine : quand celuici est élevé, il faut apporter plus de mélatonine exogène avec le traitement (Chemineau et al 1993).

\section{3 / Essais réalisés avec des implants de mélatonine (Regulin ${ }^{\circledR}$ )}

Dans tous les essais réalisés, les expérimentateurs ont cherché à réaliser un " effet bélier " (induction d'ovulations synchrones dans les jours qui suivent l'introduction des mâles, après une période de séparation d'au moins trois semaines) afin de regrouper les fécondations, donc les mise bas, sur une courte durée. Dans ce cas, le traitement avec un implant de mélatonine favorise la réponse des femelles à l'effet bélier.

Les essais australiens réalisés sur brebis Mérinos et croisées Border Leicester x Mérinos, montrent que la période optimale d'insertion de l'implant est située 6 à 3 semaines avant l'introduction des béliers dans le troupeau. Dans ce cas, l'activité ovulatoire et œstrienne débute environ un mois plus tôt dans les lots traités que dans les lots témoins,

\section{C'est la durée de sécrétion de mélatonine qui permet aux animaux de " mesurer » la durée de la nuit.}


avec un pic du taux d'ovulation très précoce équivalent à celui observé au milieu de la saison sexuelle des brebis témoins (1,6 ovulations ; Williams et al 1986). Lorsque les femelles sont mises en présence de mâles entiers, les fécondations se produisent plus tôt dans le lot traité que dans le lot témoin. De plus, la fertilité et le pourcentage de brebis mettant bas des jumeaux sont supérieurs à ceux du témoin. Au total, 15 à 20 agneaux (selon les races) pour 100 brebis traitées sont produits en plus par le lot traité (Dunstan et al 1987, Staples et al 1986, 1991).

Les essais néo-zélandais montrent que l'insertion d'un implant le 29 décembre (équivalent au 29 Juin dans l'hémisphère Nord) déclenche plus précocement l'activité sexuelle des brebis Coopworth : $78 \%$ de brebis sont saillies après un mois de lutte contre seulement $28 \%$ chez les témoins. Dans ce cas, l'avance de saison procurée par le traitement s'accompagne également d'un accroissement de la fertilité (72 vs $41 \%$ ) et de la prolificité qui conduisent à un gain de 40 agneaux pour 100 brebis traitées (McMillan et Sealey, 1989). De la même façon, en Nouvelle-Zélande également, chez des croisées Perendale et des Romney, 24 agneaux sont produits en plus pour 100 brebis traitées (Moore et al 1988).

En Grèce, les essais ont porté sur des brebis Karagouniko et East Friesland, avec l'insertion d'implants le 5 juin. Dans les deux races, l'activité sexuelle démarre plus précocement chez les brebis traitées par rapport aux brebis témoins (17 et 23 jours plus tôt respectivement) et l'étalement des venues en œstrus a été fortement réduit par le traitement ( 25 et 65 jours en moins, respectivement) (Kouimtzis et al 1989).

En Grande-Bretagne, deux larges essais cliniques ont été conduits sur un total de 2563 brebis de 3 génotypes (Mule, Scottish Blackface, Suffolk), dans 22 élevages (Haresign et al 1990). Dans ces conditions, le traitement augmente généralement la fertilité et la prolificité des brebis traitées en provoquant un déclenchement plus précoce de l'activité sexuelle. L'augmentation de la prolificité conduit à produire 15 agneaux en plus pour 100 brebis mettant bas.

La plupart de ces résultats montrent que l'utilisation d'un implant de mélatonine permet d'obtenir un déclenchement plus précoce de la saison de reproduction chez la brebis, en même temps qu'un raccourcissement de la période de lutte et qu'une amélioration de la fertilité et de la prolificité. Ce traitement permet donc, au total, un accroissement sensible du nombre d'agneaux nés par brebis mise à la lutte.

\section{4 / Intérêt de l'association ou non avec des traitements de synchronisation de l'œstrus}

Dans beaucoup d'élevages ovins français, et notamment en race Lacaune, l'utilisation de traitements hormonaux de synchronisation de l'œstrus avec éponges vaginales contenant du FGA (acétate de fluorogestone), puis injection de PMSG (gonadotrophine extraite du sérum de jument gravide, appelée maintenant eCG pour equine chorionic gonadotropin), permet à la fois la mise en place de l'insémination artificielle et l'augmentation de la productivité numérique des brebis. Toutefois, lorsque ce traitement est appliqué à contresaison ou en avance de saison, les femelles non fécondées à l'œstrus induit ne reviennent pas en chaleurs un cycle plus tard et il faut souvent attendre plusieurs semaines, voire plusieurs mois, pour que celles-ci se manifestent. Il est donc intéressant, dans les élevages français, qui sont largement utilisateurs de traitements hormonaux de synchronisation de l'œstrus (environ $18 \%$ des brebis françaises sont traitées annuellement), d'essayer de mettre en évidence le bénéfice d'une association de ceux-ci avec la mélatonine. Cette dernière pourrait, en effet, déclencher l'activité sexuelle (cyclicité) en avance de saison et donc favoriser les retours en chaleurs plus précoces chez les femelles non gravides suite au traitement hormonal d'induction.

\section{5 / Intérêt de l'association avec un traitement lumineux}

Lorsque l'on souhaite effectuer une période de fécondation en pleine contre-saison, ce qui est le cas de la forte demande actuelle dans l'espèce caprine, il est nécessaire de rétablir la sensibilité à la mélatonine, qui est en général très faible en fin d'hiver. Pour ce faire on soumet les animaux à un éclairement supplémentaire par rapport à la lumière naturelle, avant de réaliser la pose de l'implant de mélatonine. Cela nécessite un investissement qui ne s'avère intéressant que dans cette espèce où la valeur ajoutée de la lactation est importante.

Pour la production de semence en pleine contre-saison (centres d'IA), il est également nécessaire de rétablir la sensibilité à la mélatonine, en utilisant le même traitement que celui décrit ci-dessus pour les chèvres. La valeur génétique des mâles justifie la plupart du temps cet investissement. Dans ce cas, cependant, si le traitement permet une production élevée de semence de qualité à contre-saison, il n'empêche pas le retour à une période de faible activité des animaux traités en automne, quand les béliers témoins sont, eux, en pleine activité. Si l'on souhaite néanmoins avoir des mâles actifs toute l'année, on pourra utiliser l'alternance permanente d'un mois de jours longs et d'un mois de jours courts, ou d'un mois de jours longs et d'un mois de mélatonine.

Tous les essais cliniques présentés ici, sont réalisés uniquement avec des implants Mélovine®, qui ont reçu en 1995 l'autorisation de mise sur le marché en France et sont en cours d'homologation dans d'autres pays. Ces implants sont déjà d'une utilisation courante 
en Australie, en Nouvelle-Zélande, en GrandeBretagne et en Grèce, sous le nom de Regulin® (Staples et al 1991).

\section{2 / Essais réalisés en lutte naturelle dans les troupeaux ovins français}

Les objectifs des essais (lutte naturelle) ont été de tester les effets d'implants Mélovine ${ }^{\circledR}$, contenant $18 \mathrm{mg}$ de mélatonine, sur le déclenchement de l'activité sexuelle cyclique dans diverses races françaises de brebis, en lutte naturelle. Les critères susceptibles de subir une amélioration due au traitement sont donc les caractéristiques de reproduction et notamment la fertilité, la prolificité et la répartition temporelle des mise bas des animaux traités par rapport aux animaux témoins, non traités, de la même exploitation.

\section{1 / Conduite des essais}

Les expérimentations sont réalisées dans 21 élevages, regroupant 5 génotypes de brebis : la Caussenarde du Lot (8 élevages), la Limousine (1 élevage), la Tarasconnaise (6 élevages), la Rouge de l'Ouest (3 élevages) et divers croisements (3 élevages).

Dans tous les cas, c'est l'effectif total de femelles mises à la lutte à cette période de l'année qui est inclus dans l'expérimentation. Cet effectif est partagé en deux lots identiques en nombre. Les élevages dans lesquels ces essais sont mis en place sont considérés comme étant représentatifs des élevages de la race de par leur localisation géographique, leur effectif, leur système d'élevage et surtout leur mode de conduite de la reproduction. Ces élevages sont choisis par les techniciens des coopératives travaillant dans ces zones, sur les critères précédents.

Les femelles expérimentales sont des femelles considérées comme ayant des performances «normales » de reproduction antérieurement et, notamment, ayant mis-bas au moins une fois, pour les adultes, au cours de l'année précédente. Sont explicitement exclues les femelles adultes n'ayant jamais mis-bas. En outre, pour les femelles allaitantes, la mise bas doit avoir eu lieu depuis plus d'un mois lors de la pose de l'implant. Chez les agnelles, le critère de choix est essentiellement le poids vif, qui doit être estimé supérieur ou égal aux $2 / 3$ de celui des femelles adultes. D'une manière générale, tous les animaux que l'éleveur prévoyait de mettre en lutte à cette période sont inclus dans l'essai.

La mise en lots se fait en équilibrant les lots selon l'âge, la situation physiologique (agnelles, brebis sèches et allaitantes) et, dans certaines races, l'état corporel des brebis. Il est vérifié ultérieurement, avant la pose des implants, que les lots expérimentaux sont homogènes entre eux quant aux caractéristiques connues des individus, essentiellement la date de mise bas précédente.
L'implant est inséré en sous-cutané à la base de l'oreille gauche, à l'aide d'un dispositif spécial (pistolet) muni d'une aiguille (diamètre extérieur $3,5 \mathrm{~mm}$ ) dans laquelle l'implant est poussé par un piston. Aucune agrafe n'est placée après le retrait de l'aiguille qui ne provoque aucun saignement. Dans tous les cas, seules les brebis, et non les béliers, reçoivent le traitement.

L'introduction des béliers a lieu de 30 à 40 jours après la pose de l'implant. Le (ou les) bélier(s) est (sont) introduit(s) pour une durée variable (qui peut excéder 100 jours) selon les élevages, mais, pour chacun des élevages, identique entre le lot traité et le lot témoin. Dans tous les cas la lutte est libre et aucune détection des œstrus n'est effectuée.

\section{2 / Résultats}

La fertilité des femelles traitées est significativement supérieure à celle des femelles témoins : $86 \%$ vs $79 \%$, la différence est très hautement significative sur l'ensemble de l'échantillon. Dans trois races (Caussenarde, Limousine et Tarasconnaise), la fertilité des brebis traitées est significativement supérieure à celle des témoins. Dans les deux autres races, les fertilités des brebis témoins et des brebis traitées sont très élevées et très voisines (tableau 1).

La prolificité totale est également significativement supérieure chez les femelles traitées que chez les femelles témoins : 1,51 vs 1,44 agneaux par mise bas. L'augmentation de prolificité se fait surtout par la diminution des naissances simples, l'augmentation des doubles et le maintien des triples (figure 3).

La fécondité totale des brebis traitées à la mélatonine est très significativement supérieure à celle des brebis témoins non traitées : 1,30 vs 1,14 agneaux par brebis mise à la lutte, soit une augmentation de $14 \%$ dans le lot traité par rapport au lot témoin.

Compte tenu des différences entre élevages dans la date d'introduction des béliers, choisie par l'éleveur, il n'est pas possible d'effectuer une moyenne générale de date de mise bas des femelles témoins et des femelles traitées. Toutefois, l'analyse de variance qui teste l'effet élevage et l'effet traitement permet de mettre en évidence un effet très significatif de l'élevage (ce qui était attendu puisque les dates d'introduction des béliers sont très différentes entre les élevages), mais également un effet très significatif du traitement mélatonine et l'absence d'interaction significative entre élevage et traitement. Intra-élevage, la date moyenne de mise bas est plus précoce dans le lot traité que dans le lot témoin. En moyenne pour tous les élevages, la différence entre date moyenne de mise bas des femelles traitées avec la mélatonine et date moyenne de mise bas des femelles témoins est de 2,7 jours chez les Caussenardes et les Limousines, de 1,1 jours chez les Tarasconnaises et de 7 jours chez les Rouges de l'Ouest et croisées.

La durée de la période des agnelages (intervalle entre 1ère et dernière mise bas du lot 
Tableau 1. Effectif mis en lutte, fertilité, prolificité et fécondité des brebis témoins et des brebis traitées avec la mélatonine (Mélovine $®)$ pour la lutte naturelle (Chemineau et al 1991).

\begin{tabular}{|l|c|c|c|c|c|}
\hline $\begin{array}{l}\text { Race des brebis } \\
\text { (nombre d'élevages) }\end{array}$ & $\begin{array}{c}\text { Effectif } \\
\text { mis en lutte }\end{array}$ & $\begin{array}{c}\text { Fertilité } \\
\%\end{array}$ & $\begin{array}{c}\text { Nb Agneaux } \\
\text { nés }\end{array}$ & Prolificité & Fécondité \\
\hline Caussenarde (8) & 351 & 75 & 351 & 1,33 & 1,00 \\
- témoins & 347 & $83 * * *$ & 384 & 1,33 & 1,11 \\
- traitées & & & & & \\
Limousine (1) & 50 & 78 & 57 & 1,46 & 1,14 \\
- témoins & 100 & $90 * *$ & 153 & $1,70 * * *$ & $1,53 * * *$ \\
- traitées & & & & & \\
Tarasconnaise (6) & 172 & 58 & 123 & 1,24 & 0,72 \\
- témoins & 169 & $78 * * * *$ & 172 & 1,30 & $1,02 * * * *$ \\
- traitées & 185 & 89 & 281 & 1,71 & 1,52 \\
Rouge de l'Ouest (3) & 184 & 87 & 276 & 1,73 & 1,50 \\
- témoins & & & & & \\
- traitées & 173 & 98 & 250 & 1,47 & 1,44 \\
Croisées (3) & 181 & 97 & 294 & $1,68 * * *$ & $1,62 * * *$ \\
- témoins & & & & & \\
- traitées & 931 & 79 & 1062 & 1,44 & 1,14 \\
TOTAL & 981 & $86 * * * *$ & 1279 & $1,51 * * *$ & $1,30 * * *$ \\
\hline
\end{tabular}

Significativement différent du lot témoin au seuil de $*: 5 \%, * *: 2 \%, * * *: 1 \%,{ }^{*} * * *: 0,1 \%$.

considéré) est également plus courte de 2,0 jours chez les Caussenardes et les Limousines et de 28 jours chez les Rouges de l'Ouest et croisées, dans le lot traité que dans le lot témoin.

L'avance des dates moyennes de mise bas, la diminution de leur étalement dans le temps ainsi que la fertilité et la prolificité plus élevées obtenues dans les lots traités par rapport aux lots témoins apparaissent très clairement lorsque la distribution temporelle de la fécondité est représentée (figure 4).

Figure 3. Répartition des naissances selon leur mode, après la lutte naturelle chez des brebis témoins et des brebis traitées à la mélatonine (Mélovine) (d'après Chemineau et al 1991).

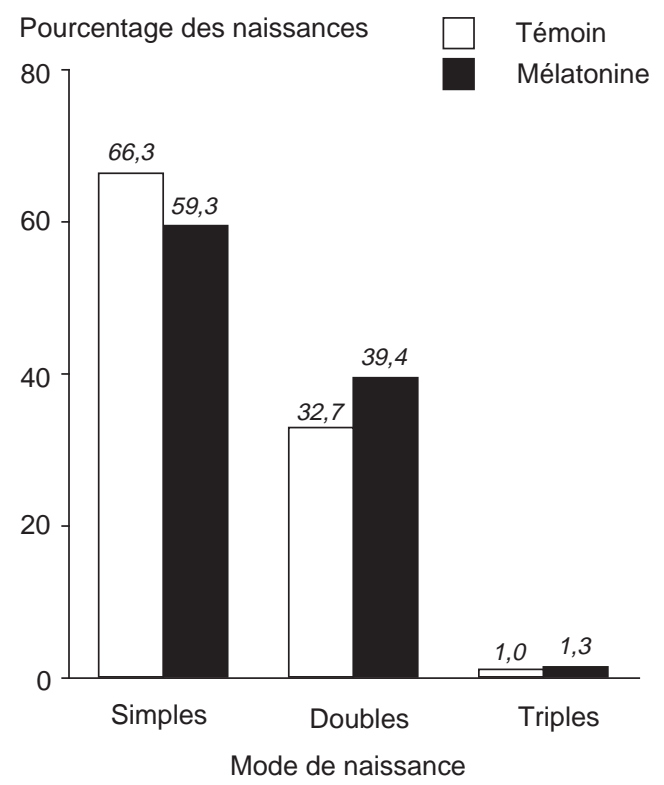

Chez les brebis Rouges de l'Ouest et croisées, le traitement par la mélatonine ne modifie ni le pourcentage d'agneaux morts nés (mélatonine 7,9\% et témoins 7,4\%), ni la mortalité dans les huit premiers jours (mélatonine $2,5 \%$; témoin $4,3 \%$ ).

Le sex-ratio à la naissance n'est pas modifié : chez les traitées ( $51 \%$ de mâles), comme chez les témoins (56\% de mâles), on observe un léger déséquilibre en faveur des mâles.

\section{3 / Essais réalisés chez la brebis, en association avec un traitement hormonal "classique " de synchronisation}

Les objectifs de ces essais ont été de tester les effets des mêmes implants Mélovine ${ }^{\circledR}$, associés à un traitement hormonal de synchronisation des œstrus, sur la fertilité et la prolificité à l'œstrus induit par le traitement et sur la fertilité et la prolificité des retours en œstrus chez les femelles non fécondées après l'IA. Il s'agit, en fait, de provoquer le déclenchement de l'activité sexuelle cyclique en avance de saison (un mois et demi avant la date normale de saison sexuelle), par induction de retours en chaleurs chez les brebis et les agnelles non fécondées (vides) après synchronisation hormonale de l'œstrus.

Les expérimentations ont été réalisées dans 9 élevages regroupant trois races de brebis : la Lacaune "Branche viande »(7 élevages), la 
Figure 4. Exemples de distribution temporelle de la fécondité cumulée (en nombre d'agneaux nés par brebis mise en lutte; $P$. Chemineau et al, non publié). Courbes rouges : brebis traitées à la mélatonine, courbes noires : brebis témoins.

Fécondité (agneaux par brebis mise en lutte)
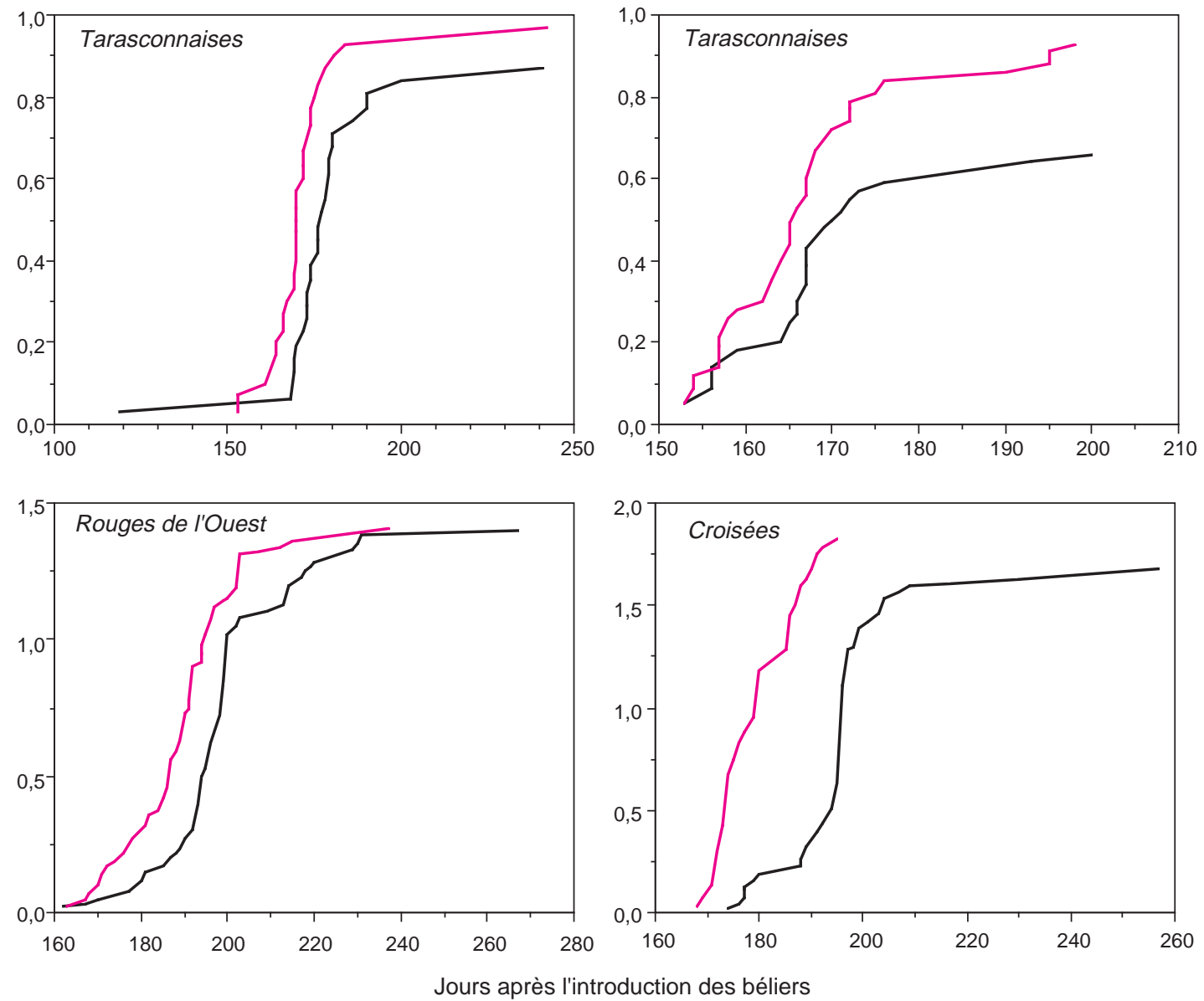

Limousine (1 élevage) et la Vendéenne (1 élevage) (tableau 2 ). Le traitement associé utilisé est le traitement classique (éponge vaginale à $30 \mathrm{mg}$ de FGA, 500 à 600 Unités Internationales de PMSG). Une seule IA est réalisée 55 \pm 1 heures après la dépose de l'éponge. L'IA a lieu en moyenne 34 jours après la pose de l'implant (31 à 41 jours selon les élevages). Les béliers utilisés pour assurer les fécondations sur retours en œstrus des femelles vides après le traitement hormonal de synchronisation, sont introduits dans la troupe après le cinquième jour qui suit l'IA. Le (ou les) bélier(s) est (sont) introduit(s) pour assurer les fécondations sur retours en œstrus, pour une durée variable selon les élevages (qui peut excéder 100 jours). Dans tous les cas la lutte est libre (lutte naturelle), aucune détection des œstrus n'est effectuée par l'éleveur. L'analyse porte sur les résultats du total œstrus induit + retours en œstrus (tableau 2).

La fertilité totale des brebis traitées avec la mélatonine est très significativement supérieure à celle des femelles témoins : 80 vs 69 $\%$. Dans la race Lacaune, la fertilité des brebis traitées est très significativement supérieure à celle des brebis témoins : 74 vs $60 \%$. Dans les deux autres races, les fertilités des brebis témoins et des brebis traitées sont très élevées et très voisines.

La prolificité des femelles traitées est significativement supérieure à celle des brebis témoins : 1,71 vs 1,59 agneaux par brebis mettant bas. Dans la race Lacaune, la prolificité est significativement supérieure chez les brebis traitées. Dans les deux autres races, la différence est faible ou inexistante.

La fécondité des brebis traitées est très significativement supérieure à celle des brebis témoins : 1,37 vs 1,09 , surtout en raison des écarts chez la Lacaune : 1,22 vs 0,89 .

\section{4 / Essais réalisés chez les caprins en association avec un traitement lumineux préalable}

Le schéma expérimenté est celui utilisant la succession dite "flash-mélatonine » (Chemineau et al 1992b). Il n'est, en effet, pas nécessaire de fournir des jours longs réels aux animaux ; l'éclairement de la phase «
Elle permet aussi d'avancer la date moyenne de mise bas et de regrouper les agnelages sur une période plus courte. 
Tableau 2. Effectif mis en lutte, fertilité, prolificité et fécondité pour le total œstrus induit + retours, par élevage, des brebis témoins et des brebis traitées avec la mélatonine (Mélovine $囚)$. Toutes les brebis (témoins et traitées) reçoivent un traitement de synchronisation (voir texte). (Chemineau et al 1991).

\begin{tabular}{|l|c|c|c|c|c|}
\hline $\begin{array}{l}\text { Race des brebis } \\
\text { (nombre d'élevages) }\end{array}$ & $\begin{array}{c}\text { Effectif } \\
\text { mis en lutte }\end{array}$ & $\begin{array}{c}\text { Fertilité } \\
\%\end{array}$ & $\begin{array}{c}\text { Nb Agneaux } \\
\text { nés }\end{array}$ & Prolificité & Fécondité \\
\hline Lacaune (7) & 203 & 60 & 179 & 1,48 & 0,89 \\
- témoins & 191 & $74 * * * *$ & 235 & $1,65 *$ & $1,22^{* * * *}$ \\
- traitées & & & & & \\
Limousine (1) & 63 & 79 & 89 & 1,78 & 1,41 \\
- témoins & 119 & 82 & 174 & 1,78 & 1,46 \\
- traitées & & & & & \\
Vendéenne (1) & 56 & 91 & 84 & 1,65 & 1,50 \\
- témoins & 105 & 86 & 155 & 1,72 & 1,48 \\
- traitées & & & & & \\
TOTAL & 322 & 69 & 352 & 1,59 & 1,09 \\
- témoins & 415 & $80 * * *$ & 564 & $1,71 *$ & $1,37 * * *$ \\
- traitées & & & & & \\
\hline
\end{tabular}

Significativement supérieur au témoin au seuil de $*: 5 \%,{ }^{* *}: 2 \%, * * *: 1 \%, * * * *: 0,1 \%$.

photosensible » (moment privilégié de la période nocturne dont l'éclairement provoque la lecture d'un jour long) est suffisant pour aboutir à la lecture d'un jour long (« JL ») et rétablir la sensibilité à la mélatonine. Dans ce cas, il est cependant nécessaire de réaliser une aube fixe par un éclairement artificiel de la chèvrerie. Lorsque l'éclairement naturel quotidien devient suffisant (puisque les animaux sont en bâtiment ouvert) l'éclairement artificiel est arrêté, puis re-démarré entre seize et dix-huit heures après cette aube fixe, soit de 22 à $24 \mathrm{~h} 00$, si l'aube fixe est réglée à 6h00. L'éclairement est apporté par des tubes fluorescents ou des lampes halogènes fournissant au moins 200 lux au niveau des yeux des animaux (Chemineau et al 1992b ; figure 5).

Dans l'espèce caprine, les résultats montrent très clairement qu'il est nécessaire d'utiliser la succession de ces deux parties du traitement pour aboutir à une activité sexuelle maximale au printemps (Chemineau et al 1986, Chemineau 1989 ; tableau 3 ). Dans ces conditions, en utilisant des boucs traités de la même façon et utilisés en lutte naturelle, la fertilité et la prolificité sont très proches, voire identiques à celles de la saison sexuelle annuelle, alors que les femelles sont fécondées en avril/mai. Le traitement permet d'obtenir une meilleure réponse à l'" effet bouc ", mais surtout d'aboutir à une cyclicité ovulatoire et œstrienne qui va conditionner les bons résultats de fertilité. Il faut remarquer néanmoins que le traitement lumineux seul permet d'aboutir à une fertilité relativement élevée.

Lorsqu'il est appliqué pendant les jours les plus courts de l'année, le traitement "JL » doit être au moins égal à deux mois. En effet, des chèvres Alpines soumises à seulement un mois de jours longs (du $5 / 1$ au 6/2) ont une fertilité significativement inférieure à celle des chèvres soumises à deux mois de "JL " (du 5/12 au 6/2) : respectivement 48 vs $71 \%$ (tableau 4 ; Chemineau 1992).

Le traitement mélatonine doit également fournir suffisamment de mélatonine pour être efficace. Ainsi, chez trois groupes de chèvres Alpines recevant trois types d'implants libérant des doses croissantes de mélatonine, la fertilité et la prolificité après lutte naturelle sont étroitement dépendantes du niveau plasmatique moyen de mélatonine : 75,77 et $97 \%$ et $1,88,2,00$ et 2,22 chevreaux par mise bas (tableau 4 ; Chemineau 1992).

Les boucs doivent recevoir le même traitement que les femelles (avec cependant une dose plus élevée par bouc). Ils peuvent être laissés à proximité de celles-ci pendant le traitement lumineux, mais doivent être impérativement séparés de tout contact avec les femelles dès la fin de la période d'éclairement supplémentaire. Il est, en effet, indispensable de réaliser un " effet bouc " pour obtenir le maximum de femelles en activité. Un ratio de 10 femelles par bouc donne généralement de bons résultats. L'introduction du bouc peut se faire entre 35 et 70 jours après la pose de l'implant. Une meilleure synchronisation semble cependant être obtenue pour un intervalle compris entre 50 et 70 jours. Lorsque l'effet mâle est réussi, le pic de fécondations a lieu entre 5 et 15 jours après l'introduction des mâles (figure 6).

Lorsqu'il est appliqué en début de lactation (ce qui n'est en général pas le cas) le traitement lumineux est susceptible d'augmenter l'ingestion alimentaire et la production laitière, alors que le traitement avec la mélatonine les diminue (Chemineau et al 1988).

Ce traitement est en cours d'essai sur un assez grand nombre d'élevages en Région Centre (URGDS du Centre) ; l'association avec un traitement classique de synchronisation, avec IA, est également testée dans plusieurs élevages de la Région Poitou-Charentes (Institut de l'Elevage). 
Figure 5. Traitements photopériodiques applicables dans les Centres d'IA ou en élevage, pour la maîtrise de l'activité sexuelle saisonnière chez les mâles et femelles des ovins/caprins.

Bâtiments fermés

(a)

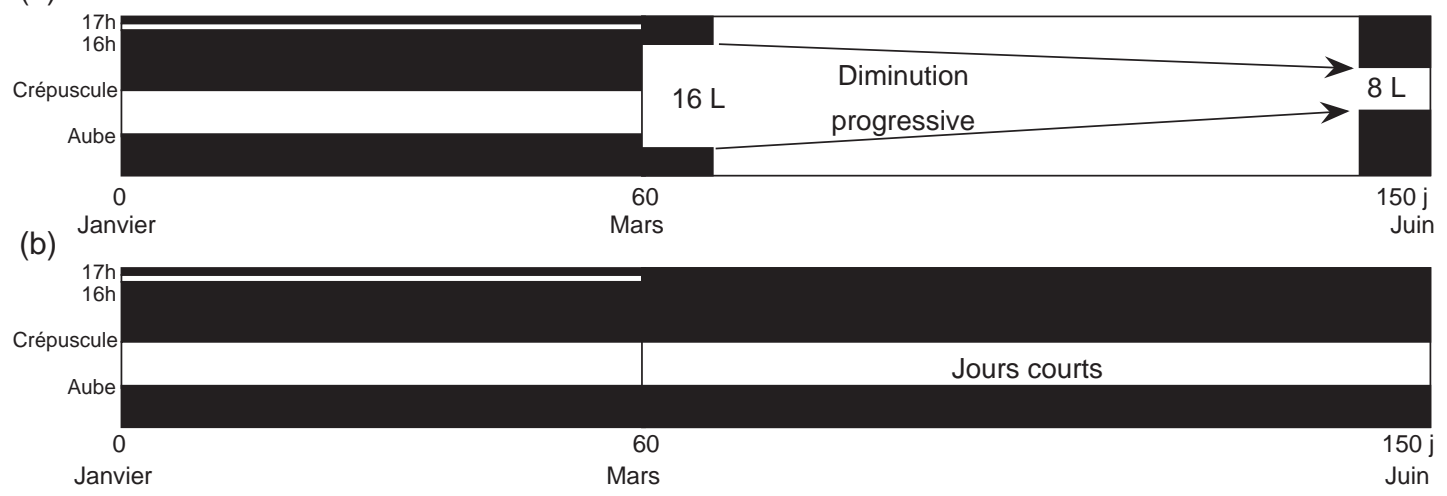

(c)

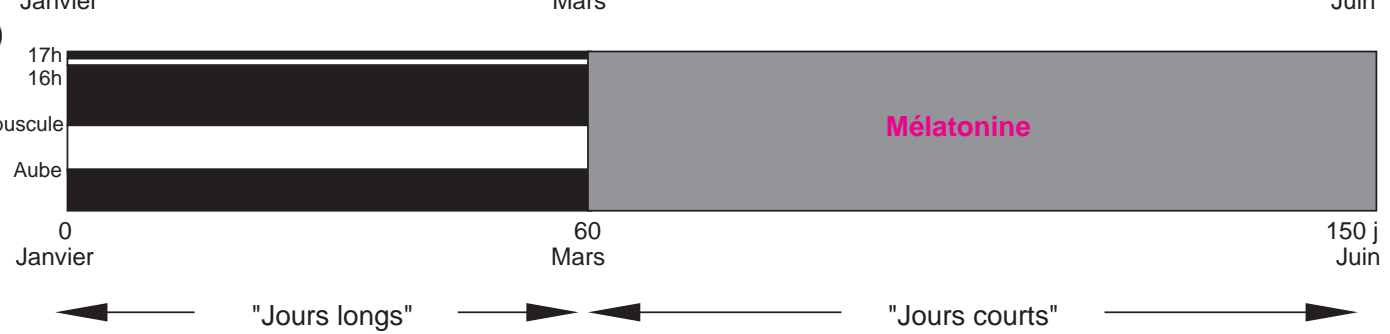

Bâtiments ouverts

(d)

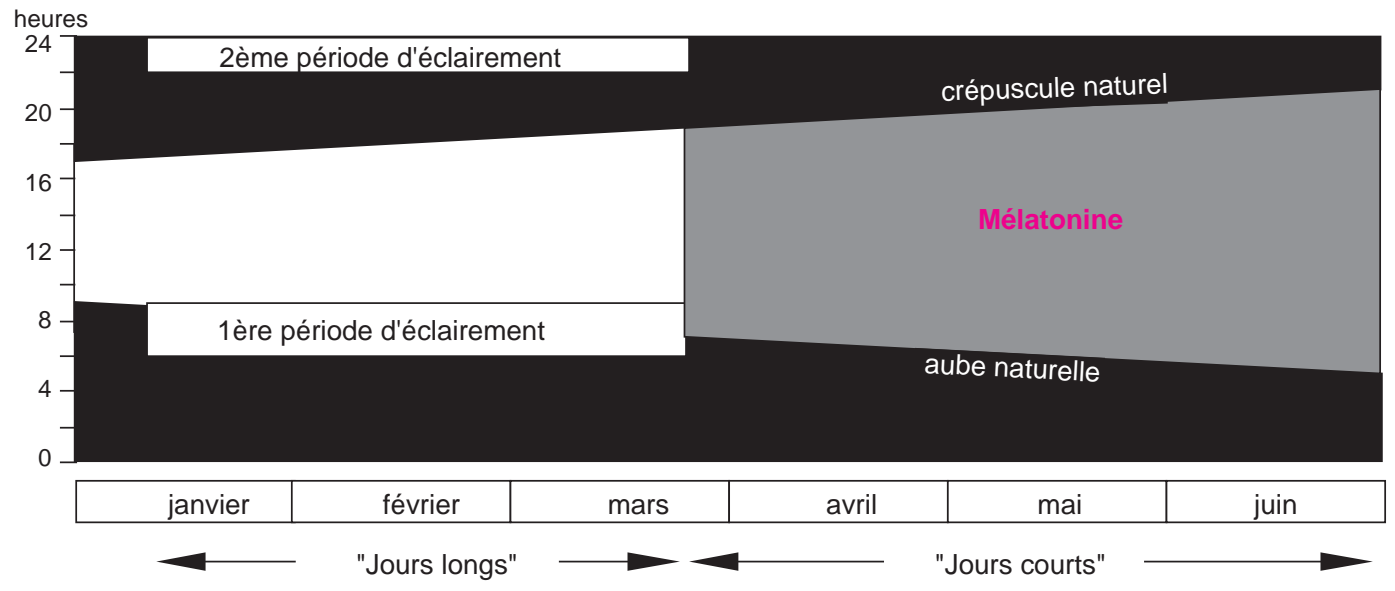

Tableau 3. Mise en évidence de la nécessité d'utiliser la succession lumière + mélatonine, pour l'obtention d'une activité sexuelle et d'une fertilité maximales à contre-saison chez la chèvre laitière française (Chemineau 1989).

\begin{tabular}{|c|c|c|c|c|}
\hline Lots & Témoin & Mélatonine & Lumière & $\begin{aligned} & \text { Lumière } \\
+ & \text { Mélatonine }\end{aligned}$ \\
\hline \multicolumn{5}{|l|}{$\begin{array}{l}\text { Expérience } 1 \text { (Adultes Saanen en lactation, } \\
\text { boucs vasectomisés du } 30 / 05 \text { au } 30 / 06 \text { ) }\end{array}$} \\
\hline Effectif & 8 & 8 & 8 & 8 \\
\hline Femelles ovulant encore 2,5 mois après l'introduction du bouc & 0 & 1 & 0 & 6 \\
\hline Femelles venues au moins deux fois en chaleurs & 2 & 5 & 5 & 8 \\
\hline \multicolumn{5}{|l|}{$\begin{array}{l}\text { Expérience } 2 \text { (Jeunes Alpines d'un an, } \\
\text { boucs entiers du } 15 / 04 \text { au 27/05) }\end{array}$} \\
\hline Effectif & 10 & 28 & 8 & 44 \\
\hline$\%$ qui ovulent pendant la lutte & 10 & 46 & 63 & 91 \\
\hline Fertilité des femelles en \% Mise Bas & 10 & 39 & 63 & 86 \\
\hline Date moyenne de fécondation & $25 / 04$ & $28 / 04$ & $03 / 05$ & $27 / 04$ \\
\hline
\end{tabular}

Chez la chèvre en contre-saison, l'activité sexuelle maximale n'est obtenue qu'en associant traitement lumineux et mélatonine. 
Tableau 4. Effet de la durée du traitement lumineux, de la dose de mélatonine et de l'intervalle pose de l'implant-introduction des boucs, sur la fertilité, la prolificité et l'intervalle moyen mâle-fécondation, chez la chèvre Alpine française. Les essais sont réalisés dans un seul élevage privé ; la date d'introduction des boucs traités varie du 15/4 au 21/5; pour les essais 2, 3, 4 et 5 les chèvres ont reçu au moins 60 jours de " $J L$ », pour les essais 3,4 et 5, les chèvres ont reçu la dose 3 de mélatonine. Dans l'essai 4, les boucs sont introduits à la même date, dans l'essai 5, l'introduction des boucs est décalée de 16 jours. (P. Chemineau, non publié).

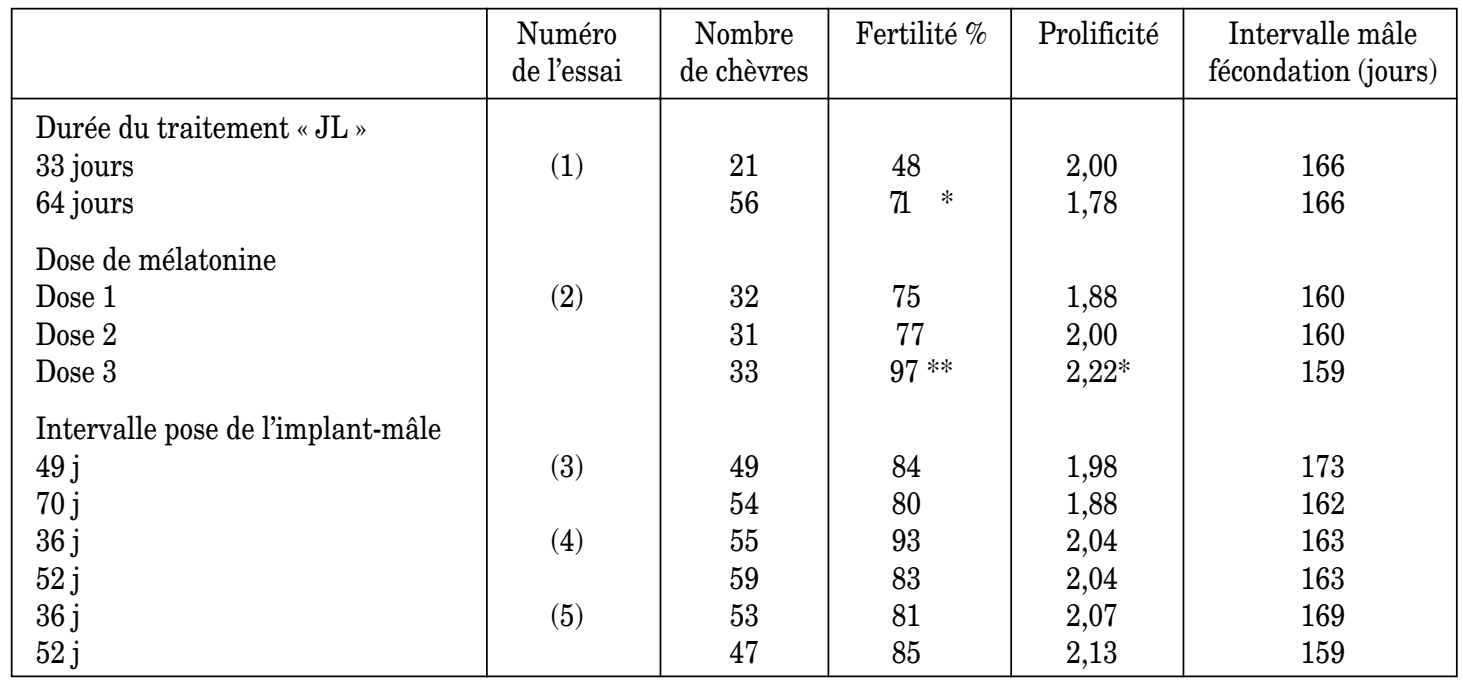

Significativement différent au seuil de $*: 5 \%, * *: 1 \%$.

Figure 6. Distribution des mise bas après l'introduction du bouc chez la chèvre Alpine française traitée avec la succession de 2 mois de " $J L$ » et d'un implant de mélatonine. L'introduction des boucs, ayant reçu le même traitement, a lieu 36 ou 52 jours après la pose de l'implant. L'expérience est réalisée deux années consécutives : 1992 (introduction des boucs le 17/4) et 1993 (introduction des boucs le 21/5). (P. Chemineau, non publié).

Pourcentage de mise bas



\section{5 / Essais réalisés chez les béliers}

Chez le bélier Ile-de-France recevant deux mois d'éclairement supplémentaire (une heure d'éclairement nocturne 16 à 17 heures après une aube fixe ; figure 5) suivis de l'insertion d'un implant de mélatonine, le poids testiculaire augmente au bout de quelques semaines pour devenir significativement supérieur à celui des béliers témoins pendant une durée voisine de 100 jours (Chemineau et al 1992a ; figure 7). Cette augmentation du poids testiculaire s'accompagne d'une amélioration du comportement sexuel à la collecte et de la quantité et qualité des spermatozoïdes produits, par rapport aux béliers témoins (Hanif et Williams 1991). Le nombre de doses par saut est très significativement augmenté dans le lot traité par rapport au lot témoin (10,5 vs 7,9 ; Fidelle et Arranz 1993). De plus, la fertilité de la semence utilisée en IA est supérieure chez les béliers traités par rapport aux béliers témoins (67,6 vs $56,0 \%$ sur 198 et 200 brebis inséminées artificiellement dans les mêmes troupeaux ; Chemineau et al 1992a).

Ce type de traitement, utilisant la mélatonine ou en substitution trois mois de jours décroissants (figure 5), est aussi utilisable chez les jeunes béliers pour pouvoir avancer la reprise de croissance testiculaire et ainsi les utiliser pour produire de la semence avant leur première année d'âge (Colas et al 1987). Tous les jeunes mâles des deux schémas de sélection de la race Lacaune sont actuellement traités de cette manière (plus de 500 jeunes béliers traités par an).

L'alternance d'un mois de jours courts et d'un mois de jours longs a été décrite comme permettant d'éviter l'installation d'un état réfractaire et donc permettant de maintenir une activité spermatogénétique élevée et constante au cours du traitement, qui peut être appliqué pendant plusieurs années consécutives (Pelletier et al 1987). Dans cette situation, les animaux produisent toute l'année une semence en quantité et en qualité au moins égale à celle produite pendant la saison sexuelle annuelle par des animaux non traités (Chemineau et al 1988). Dans les conditions d'un centre d'IA, sur plusieurs races, l'application de cette alternance rapide d'un mois de jours courts et d'un mois de jours longs a permis de plus que doubler la production de spermatozoïdes utiles (de qualité suffisante pour être utilisés en IA) par rapport aux béliers témoins non traités du même centre (Chemineau et al 1992a). Ce traitement n'est cependant applicable que dans des bâtiments fermés et ventilés, ce qui limite son utilisation aux centres de production de semence disposant de certains moyens financiers et techniques.

Pour tester l'efficacité de ce schéma dans des conditions de bâtiment ouvert, c'est-àdire lorsque les animaux perçoivent les variations naturelles de la photopériode, plusieurs essais ont été réalisés. Les deux premiers, qui se sont avérés infructueux, ont consisté l'un en l'alternance d'un mois où une heure d'éclairement nocturne était imposé, avec un mois de photopériode naturelle, et l'autre en

Figure 7. Poids testiculaire moyen de béliers lle-de-France témoins et traités avec la succession " Jours longs » + implants de mélatonine (Chemineau et al 1992a).

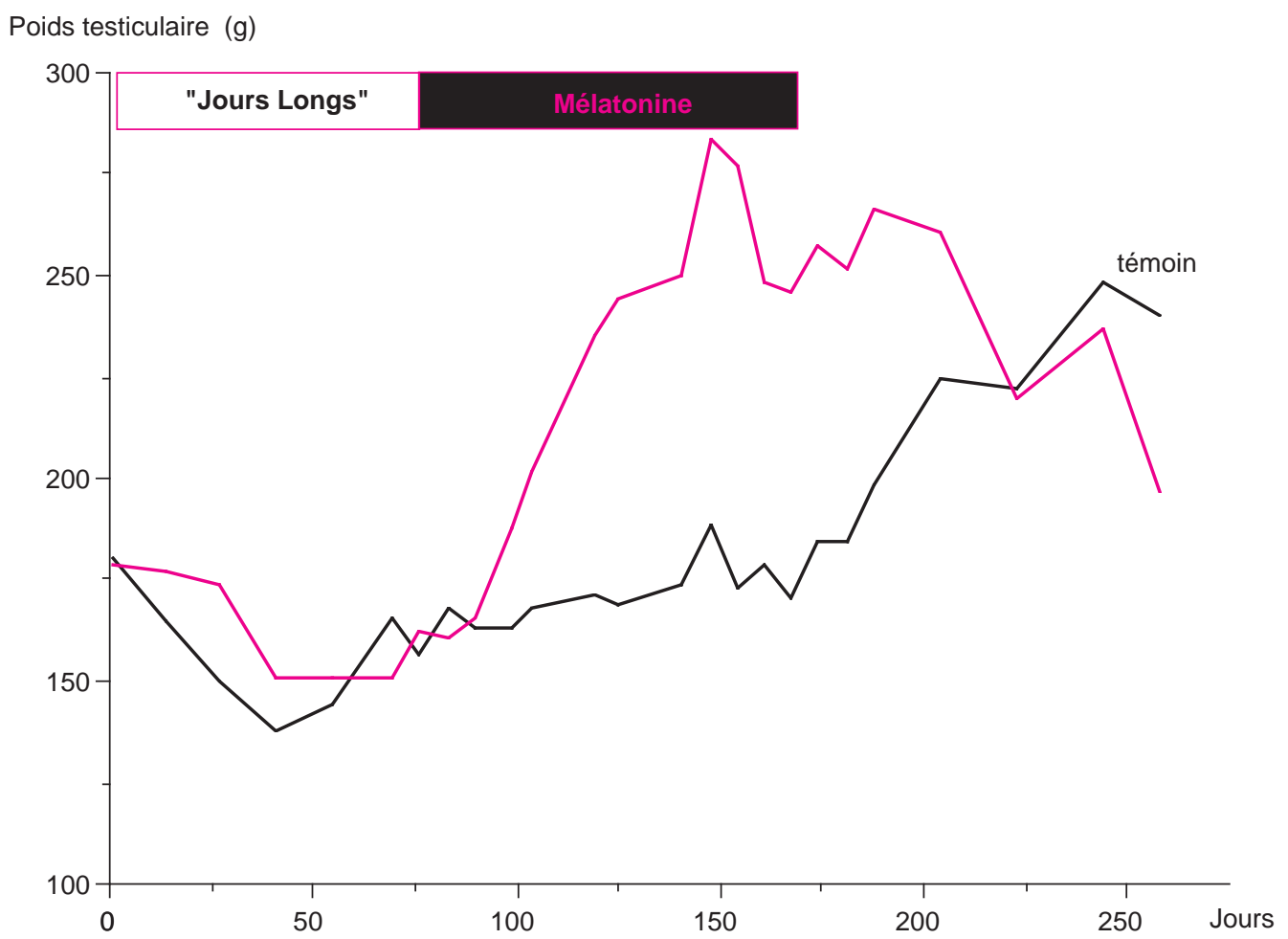

Chez le bélier soumis à 2 mois d'éclairement supplémentaire, le traitement mélatonine augmente le poids testiculaire, qui reste supérieur à celui des témoins pendant environ 100 jours. 
Figure 8. Evolution du poids testiculaire moyen de béliers lle-de-France, témoins ou traités avec une alternance d'un mois d'une heure d'éclairement artificiel nocturne avec un mois d'implant sous-cutané de mélatonine (Malpaux et al 1993a).

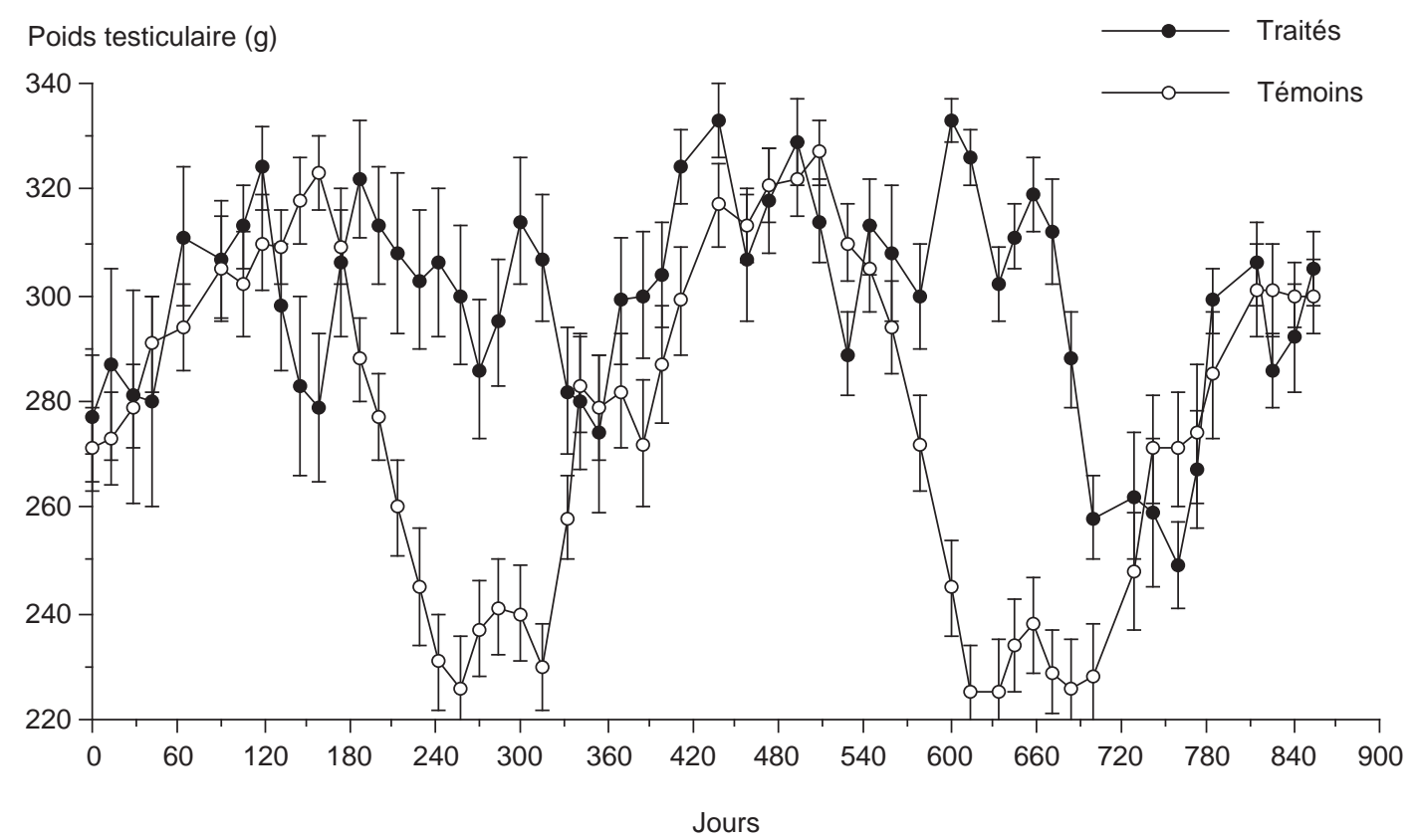

l'alternance d'un mois d'implant de mélatonine avec un mois d'éclairement naturel. Le dernier essai a consisté en l'alternance d'un mois d'éclairement nocturne et d'un mois d'implant de mélatonine. Dans ces conditions, le poids testiculaire des béliers Ile-de-France traités reste constamment élevé pendant presque deux ans, alors que celui des béliers témoins conduits dans les mêmes conditions d'élevage subit les variations habituelles (figure 8 ; Malpaux et al 1993a). En fin d'expérience cependant, le poids testiculaire des béliers traités diminue pour retrouver les valeurs des béliers témoins, ce qui pourrait être dû à la difficulté pratique de retrait des implants des oreilles des animaux, de façon répétitive à la fin de chaque période de mélatonine. Ce traitement reste à perfectionner sur ce point, mais la démonstration de son efficacité en conditions d'éclairement naturel est faite.

\section{6 / Essais réalisés chez les boucs}

De la même façon que chez le bélier, l'alternance rapide entre jours longs et jours courts entraîne chez le bouc l'abolition ou l'atténuation des variations saisonnières de l'activité sexuelle.

Ainsi, une alternance d'un mois de jours courts et d'un mois de jours longs, ou de deux mois de jours courts et de deux mois de jours longs, permet d'éviter la baisse saisonnière $\mathrm{du}$ poids testiculaire et de la production spermatique observée chez les témoins et ce pendant les trois années d'application des traitements, chez des boucs soumis à un rythme de collecte moyen (deux éjaculats par semaine) (figure 9). Malgré l'existence de certains problèmes comportementaux (agressivité excessive, augmentation du temps de latence à la collecte), les boucs subissant ces alternances rapides produisent, au cours des deux premières années de traitement, de 55 à $69 \%$ plus de doses d'IA que les boucs témoins non traités (Delgadillo et al 1992, 1993). Mesurée intra-troupeaux sur plus de 1500 chèvres, la fertilité de la semence congelée collectée des boucs des trois lots n'est pas significativement différente entre lots (tableau 5). La collecte intensive de ces boucs après trois ans de traitement, montre que leur production spermatique quotidienne est supérieure de $24 \%$ (1 mois $/ 1$ mois $)$ et $111 \%$ ( 2 mois $/ 2$ mois $)$ à celle des boucs témoins (Delgadillo et al 1993).

Dans un rythme de collecte plus intensif (4 collectes par semaine tout au long de l'année), des boucs soumis à l'alternance deux mois de jours courts/deux mois de jours longs, produisent $41 \%$ de doses d'IA en plus que des boucs maintenus en photopériode naturelle et collectés selon le rythme habituel des professionnels (4 éjaculats par semaine, de septembre à février). La fertilité de la semence produite par les boucs ainsi traités est inférieure à celle produite par les boucs témoins (tableau 5).

Ce schéma photopériodique est actuellement en cours d'utilisation par Capri-IA (centre coopératif de production de semence caprine).

\section{Discussion / Conclusion}

En lutte naturelle, la fertilité des brebis traitées avec Mélovine $\AA$ est améliorée par 
rapport à celle des femelles témoins. Le déclenchement des fécondations se fait plus précocément dans le lot traité que dans le lot témoin et ceci de façon plus groupée puisque la durée totale des agnelages est réduite dans le lot traité par rapport au témoin. Cette observation est intéressante car elle montre que le traitement permet le déclenchement plus précoce de l'activité sexuelle ainsi que la réduction de la durée des agnelages ce qui est un avantage non négligeable en élevage ovin.

La prolificité est améliorée dans le lot traité par rapport au lot témoin. Il faut remarquer que cet accroissement de prolificité se fait par l'intermédiaire d'une diminution du nombre de naissances simples et d'une augmentation du nombre de naissances doubles sans modification de celui de naissances triples. Ce résultat est important pour l'éleveur qui, dans tous les cas, souhaite augmenter la prolificité de ses brebis sans augmenter le nombre de naissances de triplés qui l'obligent à avoir recours, presque obligatoirement, à l'allaitement artificiel.

La fécondité est donc accrue dans le lot traité par rapport au lot témoin et c'est une augmentation de 16 agneaux nés pour 100 brebis traitées qui est obtenue grâce au traitement.

Ces résultats sont, dans leur ensemble, tout à fait comparables à ceux obtenus par plusieurs auteurs (Staples et al 1986, Williams et al 1986, Moore et al 1988, Mc Millan et Sealey 1989, Haresign et al 1990), dans

Figure 9. Poids testiculaire moyen et production spermatique de boucs Alpins et Saanen, témoins (maintenus en photopériode naturelle) ou traités (alternance d'un mois de jours longs et d'un mois de jours courts), au cours de trois années consécutives (d'après Delgadillo et al 1992, 1993).

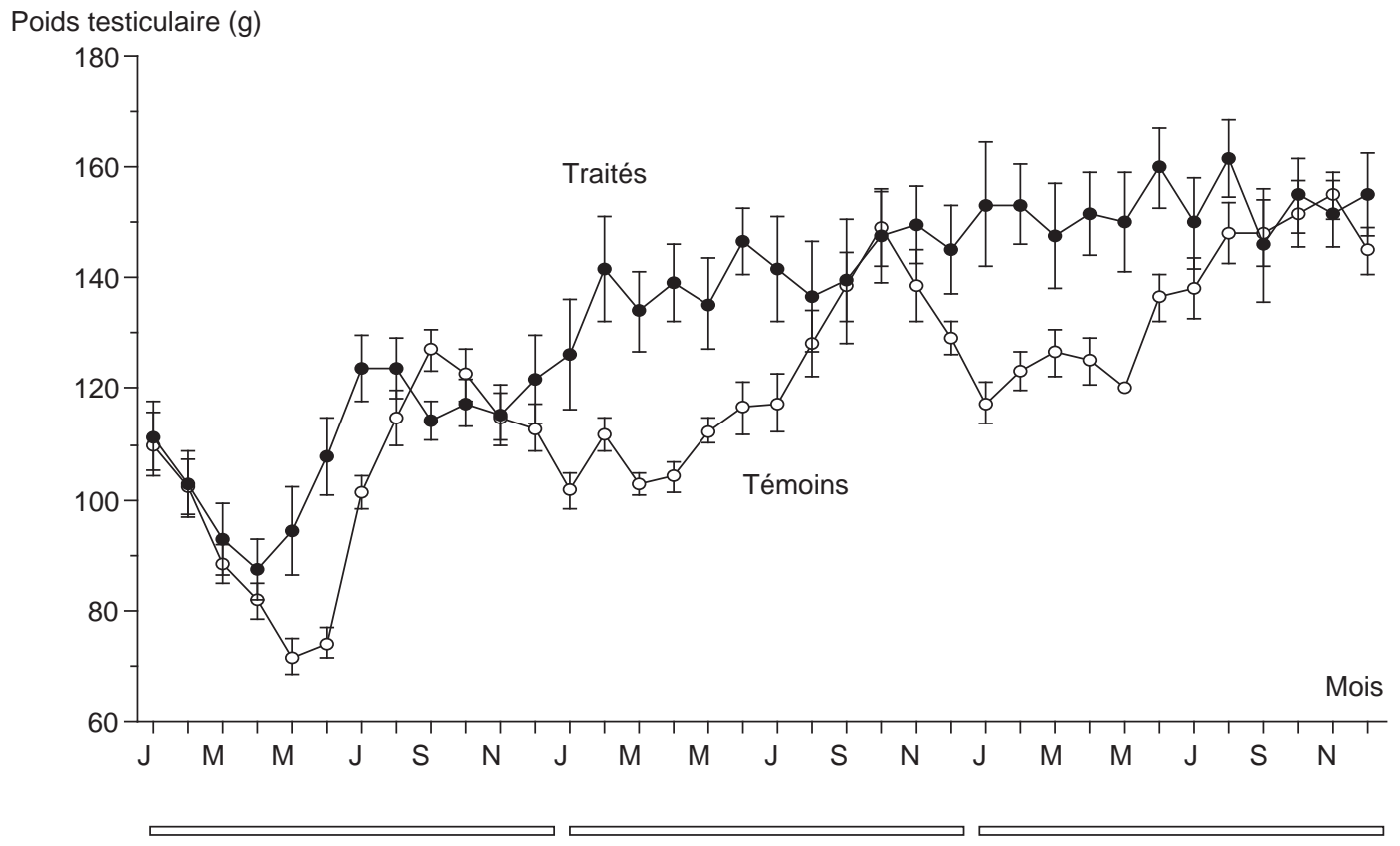

L'alternance d'un mois de jours courts et un mois de jours longs permet d'éviter la baisse saisonnière du poids testiculaire et de la production spermatique.

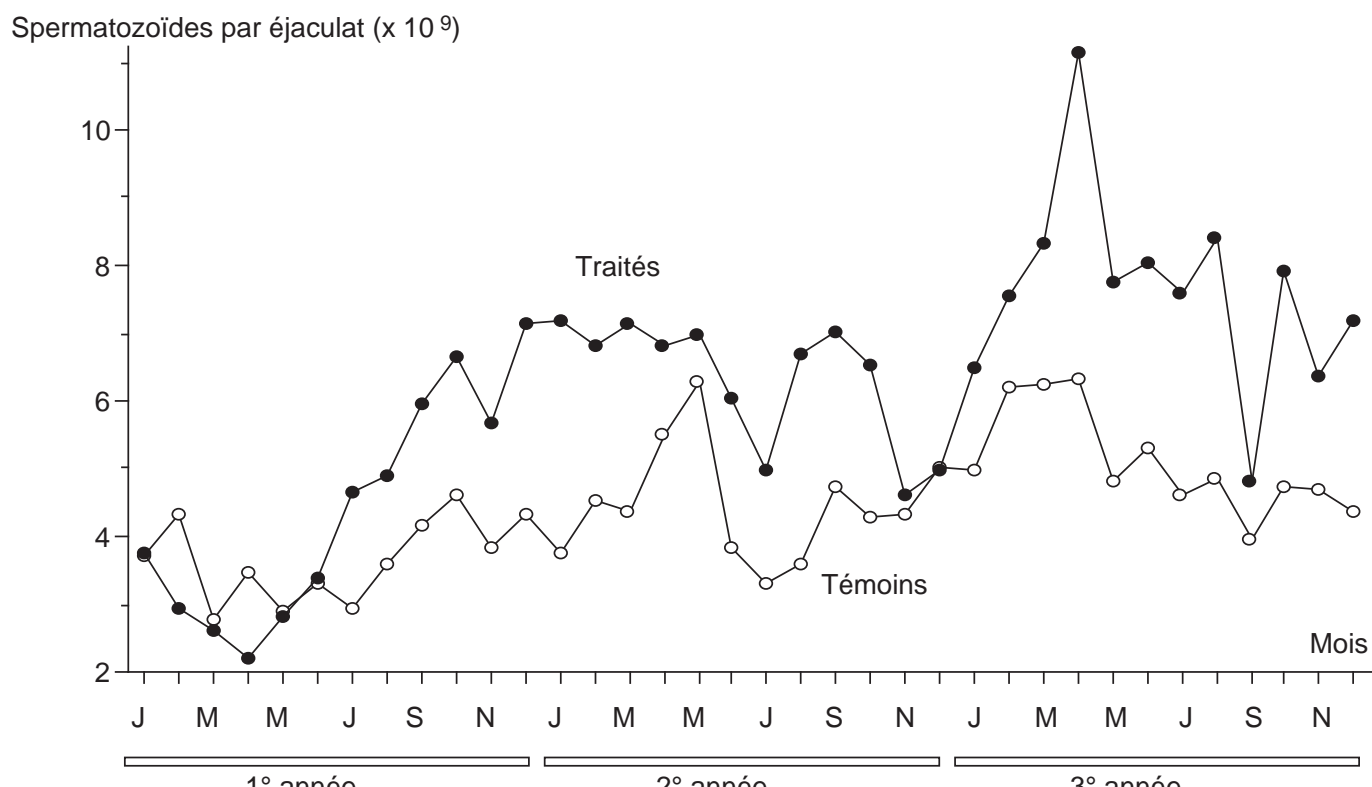


Tableau 5. Fertilité après IA (expulsions constatées) de la semence de boucs témoins (soumis à l'éclairement naturel) ou traités par une alternance rapide de jours longs (16 heures de lumière par 24 heures) et de jours courts (8 heures de lumière par 24 heures). Les IA sont réalisées dans chaque élevage, après répartition des femelles à inséminer dans chacun des lots de boucs traités et/ou témoins.

\begin{tabular}{|l|c|c|c|}
\hline Lots expérimentaux & $\begin{array}{c}\text { Témoins } \\
\text { (éclairement naturel) }\end{array}$ & $\begin{array}{c}\text { 1 mois JL } \\
\text { 1 mois JC }\end{array}$ & $\begin{array}{c}2 \text { mois JL } \\
2 \text { mois JC }\end{array}$ \\
\hline $\begin{array}{l}\text { Expérience 1 (Delgadillo et al 1992) } \\
\text { (1 599 chèvres également réparties dans 58 troupeaux) }\end{array}$ & & & \\
Nombre de boucs & 6 & 6 & 6 \\
Rythme de collecte (nb d'éjaculats / semaine) & 2 & 2 & 2 \\
Nombre de doses produites par an & 253 & 427 & 391 \\
Fertilité (\% expulsions constatées) & 62,5 & 57,9 & 57,8 \\
Expérience 2 (B. Lebouf et P. Chemineau, non publié) & & & \\
(785 chèvres également réparties dans 25 troupeaux) & & & 6 \\
Nombre de boucs & 6 & & 4 toute l'année \\
Rythme de collecte (nb d'éjaculats / semaine) & 4 de sept. à février & & 9333 \\
Nombre de doses produites par an & 6637 & & 61,2 \\
Fertilité (\% expulsions constatées) & 69,5 & & \\
\hline
\end{tabular}

d'autres races de brebis pour lesquelles une avance de la saison sexuelle et un accroissement de la fécondité sont obtenus après traitement avec les mêmes implants.

Pour le total œstrus induit + retours, la différence entre les lots traités et les lots témoins, est importante pour les trois paramètres étudiés. La fertilité est accrue de 11 points (soit $+16 \%$ par rapport au témoin), la prolificité de 12 agneaux pour 100 brebis mettant bas (soit $+7 \%$ par rapport au témoin) et la fécondité de 30 agneaux pour 100 femelles mises en reproduction (soit $+25 \%$ par rapport au témoin).

Chez les caprins, l'association traitement lumineux et mélatonine, puis " effet bouc », induit une activité cyclique en pleine contresaison, qui conduit à l'obtention d'une fertilité élevée, proche de celle observée en saison sexuelle. Les essais actuellement en cours permettront de préciser l'intérêt et les limites de ce type de traitement.

Chez le bélier, si l'utilisation d'implants seuls est souhaitable dans le cas où les brebis d'un élevage sont traitées, l'association avec un traitement lumineux préalable permet l'induction d'une activité spermatogénétique élevée en pleine contre-saison. L'utilisation d'une alternance rapide entre un mois de jours courts et un mois de jours longs conduit à une suppression totale des variations saisonnières et à une augmentation spectaculaire de la quantité et de la qualité de la semence des béliers et des boucs ainsi traités.

\section{Remerciements}

Les auteurs souhaitent remercier F. Maurice, A. Daveau, D. Chesneau, Y. Guérin, L. Brunet, G. Durand (INRA Nouzilly), Y. Berson et J. Bernelas (INRA SEIA), L.D. Staples (Applied Biotechnologies Ltd), Y. Cognié (INRA Nouzilly), C. Jardon et B. Broqua (Institut de l'Elevage), P. Fauconnet (Limousine Coop), H. Issaly, J.P. Vaur (EDE du Lot), J.P. Belloc (Ovitest), M. Briois (Confédération des Prod. de lait de brebis et de fromage de Roquefort) et J.M. Arranz (CIOP Pyrénées Atlantiques) pour leur aide. Les expérimentations ont reçu le concours financier de SANOFI Santé Nutrition Animale, de la Région Centre et du Critt ISIS. Les expérimentations conduites dans le rayon de Roquefort et dans les Pyrénées Atlantiques ont été conduites dans le cadre du groupe technique "reproduction » du CNBL. Les implants ont été fournis par Hœchst UK.

\section{Références bibliographiques}

Arendt J., Symons A.M., Laud C.A., Pryde S.J., 1983. Melatonin can induce the early onset of the breeding season in ewes. J. Endocrinol., 97, 395-400.

Bittman E.L., Dempsey R.J., Karsch F.J., 1983. Pineal melatonin secretion drives the reproductive response to daylength in the ewe. Endocrinology, 113, 2276-83.

Chemineau P., 1989. Le désaisonnement des chèvres par la lumière et la mélatonine. La Chèvre, 171, 1822.

Chemineau P., 1992. Seasonality and photoperiodic influence in the female goat reproduction. Vth International Conference on goats, New Delhi, Volume II, Part II, 355-368.
Chemineau P., Normant E., Ravault J.P., Thimonier J., 1986. Induction and persistence of pituitary and ovarian activity in the out-of-season lactating dairy goat after a treatment combining a skeleton photoperiod, melatonin and the male effect. Journal of Reproduction and Fertility, 78, 497-504.

Chemineau P., Pelletier J., Guérin Y., Colas G., Ravault J.P., Touré G., Almeida G., Thimonier J., Ortavant R., 1988. Photoperiodic and melatonin treatments for the control of seasonal reproduction in sheep and goats. Reproduction Nutrition Développement (Colloque International : "Mécanismes neurœndocriniens et contrôle photopériodique de la reproduction chez les animaux domestiques ". 17-18 Septembre 1987, Nouzilly (France)) 28, 409-422. 
Chemineau P., Vandaele E., Brice G., Jardon C., 1991. Utilisation des implants de mélatonine pour l'amélioration des performances de reproduction chez la brebis. Recueil de Médecine Vétérinaire, Spécial Reproduction des Ruminants, Mars-Avril, 227-239.

Chemineau P., Malpaux B., Delgadillo J.A., Guérin Y., Ravault J.-P., Thimonier J., Pelletier J., 1992a. Control of sheep and goat reproduction : use of light and melatonin. Animal Reproduction Science, 30, 157-184.

Chemineau P., Malpaux B., Guérin Y., Maurice F., Daveau A., Pelletier J., 1992b. Lumière et mélatonine pour la maîtrise de la reproduction des ovins et des caprins. Annales de Zootechnie, 41, 247-261.

Chemineau P., Maurice F., Daveau A., 1993. Re-initiation of ovulatory activity by melatonin given as a constant-release implant in long-day treated Ile-deFrance ewes depends on endogenous secretion of melatonin. Dans Melatonin and the pineal gland.From basic science to clinical application. Y. Touitou, J. Arendt et P. Pévet, eds, Elesvier Science Publ, 247-250.

Colas G., Guérin Y., Briois M., Ortavant R., 1987. Photoperiodic control of testicular growth in the ram lamb. Animal Reproduction Science 13, 255-262.

Collin J.P., Arendt J., Gem W., 1988. Le "troisième œil ». La Recherche, n 203, Volume 19, 1154-1165.

de Reviers M.M., Ravault J.P., Tillet Y., Pelletier J., 1989. Melatonin binding sites in the sheep pars tuberalis. Neuroscience Letters, 100, 89-93.

Delgadillo J.A., Lebœuf B., Chemineau P., 1992. Abolition of seasonal variations in semen quality and maintenance of sperm fertilizing ability by short photoperiodic cycles in he-goats. Small Ruminant Research, 9, 47-59.

Delgadillo J.A., Lebœuf B., Chemineau P., 1993. Maintenance of sperm production in bucks during a third year of short photoperiodic cycles. Reproduction Nutrition Développement, 33, 609-617.

Dunstan E., McPhee S., Williams A., Staples L., 1987. Time of melatonin treatment in relation to ram introduction for optimum performance of ewes joined in spring and summer. Proceedings Australian Society Animal Production, 17, 391 (Abstract).

English J., Poulton A.L., Arendt J., Symons A.M., 1986. A comparison of the efficiency of melatonin treatments in advancing œstrus in ewes. Journal of Reproduction and Fertility, 77, 321-327.

Fidelle F., Arranz J.M., 1993. Traitement lumineux des antenais et des agneaux de la CIOP. SicaCREOM, Ordiarp.

Hanif M., Williams Ll., 1991. The effects of melatonin and light treatment on the reproductive performance of yearling Suffolk rams. British Veterinary Journal, 147, 49-56.

Haresign W., Peters A.R., Staples L.D., 1990. The effect of melatonin implants on breeding activity and litter size in commercial sheep flocks in the UK. Animal Production, 50, 111-121.

Karsch F.J., Bittman E.L., Foster D.L., Goodman R.L., Legan S.J., Robinson J.E., 1984. Neurœndocrine basis of seasonal reproduction. Recent Progress in Hormone Research, 40, 185-232.

Kennaway D.J., Gilmore T.A., Seamark R.E., 1982. Effect of melatonin feeding on serum prolactin and gonadotropin and the onset of seasonal œstrous cyclicity in sheep. Endocrinology, 110, 1766-1772.
Kouimtzis S.A., Belibasaki S., Doney J.M., 1989. Melatonin advances and condenses the onset of seasonal breeding in greek dairy ewes. Animal Production, 48, 399-405.

Malpaux B., Chemineau P., Pelletier J., 1993a. Melatonin and reproduction in sheep and goats. In Melatonin, biosynthesis, physiological effects and clinical applications. H.S. Yu and R.J. Reiter eds, CRC Press Boca Raton Publishers, 253-287.

Malpaux B., Daveau A., Maurice F., Gayrard V., Thiéry J.C., 1993b. Short-day effects of melatonin on LH secretion in the ewe : evidence for central sites of action in the mediobasal hypothalamus. Biology of Reproduction, 48, 752-760.

Malpaux B., Daveau A., Maurice F., Locatelli A., Thiéry J.C., 1994. Evidence that melatonin binding sites in the pars tuberalis do not mediate the photoperiodic actions of melatonin on $\mathrm{LH}$ and prolactin secretion in ewes. Journal of Reproduction and Fertility, 101, 625-632.

McMillan W.H., Sealey R.C., 1989. Do melatonin implants influence the breeding season in Coopworth ewes ? Proceedings New Zealand Society of Animal Production, 49, 43-45.

Moore R.W., Miller C.M., Dow B.W., Staples L.D., 1988. Effects of melatonin on early breeding of $\mathrm{F}+$ and ++ Booroola x Perendale and Romney ewes. Proceedings New Zealand Society of Animal Production, 48, 109-111.

Mori Y., Shimizu K., Hoshino K., 1987. A rise in peripheral melatonin levels induces ovarian activity in anœstrous sheep. Japanese Journal of Animal Reproduction, 33, 155-159.

Nett T.M., Niswender G.D., 1982. Influence of exogenous melatonin on seasonality of reproduction in sheep. Theriogenology, 17 (6), 645-653.

Nowak R., Rodway R.G., 1985. Effect of intravaginal implants of melatonin on the onset of ovarian activity in adult and prepuberal ewes. Journal of Reproduction and Fertility, 74, 287-293.

Nowak R., Rodway R.G., 1987. Length of melatonin exposure and onset of ovarian activity in anœstrus ewes. Journal of Reproduction and Fertility, 80, 343347.

Pelletier J., Chemineau P., Thimonier J., VollandNail P., 1987. Environment and Changes in Reproductive Activity in Sheep and Goats. P. Pévet ed., Comparative Physiology of Environmental Adaptations, vol. 3. 8th ESCP Conf., Strasbourg 1986, Karger, Basel (publ.), 121-135.

Poulton A.L., Symons A.M., Kelly M.I., Arendt J., 1987. Intraruminal soluble glass boluses containing melatonin can induce early onset of ovarian activity in ewes. Journal of Reproduction and Fertility, 80, 235-239.

Ravault J.P., Thimonier J., 1988. Melatonin patterns in ewes maintained under skeleton or resonance photoperiodic regimens. Reproduction Nutrition Développement, 28(2B), 335-540.

Ronayne E., Jordan B., Quirke J.F., Roche J.F., 1989. The effect of frequency of administration of melatonin on the time of onset of the breeding season in anœstrus ewes. Animal Reproduction Science, 18, 13-24.

Staples L., McPhee S., Ayton B., Reeve J., Williams A., 1986. Optimum melatonin treatment to improve reproductive performance of Merino, Border Leicester $\mathrm{x}$ Merino and Rommey ewes joined in spring and 
early summer. Proceedings Australian Society of Reproductive Biology, 18, 26 (Abstract).

Staples L., McPhee S., Reeve J., Williams A.H., 1991. Practical applications for controlled release melatonin implants in sheep. Advances in pineal research : 6. A. Foldes, R.J. Reiter eds, J. Libbey \& Co Ltd publ., 199-208.

Stellflug J.N., Nett T.M., Parker C.F., 1988. Melatonin advances june breeding and fall lambing in range and farm-flock type ewes. Theriogenology, 29 (3), 643-655.
Thimonier J., 1989. Contrôle photopériodique de l'activité ovulatoire chez la brebis. Existence de rythmes endogènes. Thèse de Doctorat Sciences de la Vie. Université de Tours, 112 pp.

Williams A.H., McPhee S., Ayton B.M., Staples L.D., 1986. Dose dependent effects of continuous melatonin administration on the seasonal pattern of œstrous cyclicity and ovulation rate in corriedale ewes. Proceedings Australian Society of Reproductive Biology, 18, 27 (Abstract).

\begin{abstract}
Use of melatonin implants and photoperiodic treatments to control seasonal reproduction in sheep and goats.

Melatonin is a natural compound naturally secreted by the pineal gland during the dark phase of the light/dark cycle, which transduces the photoperiodic information in mammals. The continuous administration of melatonin via a subcutaneous implant, as described here, allows to mimic short days while animal's eyes perceive long days of spring and summer. It is necessary to impose an alternation between long and short days in order to control the period of sexual activity.

In ewes under natural mating, a subcutaneous implant of melatonin (Melovine ${ }^{\circledR}$ ) is inserted from 30 to 40 days before joining. The various experiments realised since some years in 5 French sheep breeds, and which compared in the same flocks treated and control ewes, have shown that fecundity of treated ewes is much higher than that of control ewes (16 lambs more per 100 ewes joined). Dates of lambing are more precocious and less spread in treated than in control ewes. Always in the ewe, in association

breeding season. The association with « classical " hormonal treatments and $\mathrm{AI}$ is on test.

In the ram, insertion of melatonin implants allows an advance of testicular growth and an improvement of sperm production. If a full outof-season sperm production is wished, as in AI centres, it is necessary previously to the insertion of implants to impose a light treatment composed of real long days or long days mimicked by one hour of extra light during the photosensitive phase. Such a treatment stimulates testicular growth, improves sexual behaviour, sperm production and increases fertility after AI, when compared to control rams. It is also possible to impose an alternation of one month of long days and one month of short days, which induces the maintenance of an elevated and constantly high sperm production during consecutive years. In open barns, in males left under natural lighting, the alternation of one month of extra light during the night with one month of melatonin treatment allows to reach the same results, removal of implants at the end of treatment remaining, however, difficult.
\end{abstract} with "classical " hormonal treatments for the control of oestrus and artificial insemination, melatonin treatment allows to significantly increase fecundity for the total induced oestrus and returns (30 lambs born more compared to control ewes).

In the dairy goat, due to the strong demand existing for an out-of-season breeding (from April to July), it is recommended to impose a photoperiodic treatment (extra light with a fixed dawn and a nocturnal lighting) during more than 2 months before implant insertion. Natural mating is done by introducing treated bucks among the female goats, from 35 to 70 days after the end of the photoperiodic treatment, in order to use the " buck effect ". Under these conditions, fertility is close to that normally observed during the annual breeding season (higher than $80 \%$ ) and fertilisations occur about 10 days after introduction of bucks. Litter size is close to that of the normal
In the buck, the same type of rapid alternation between long days and short days allows to maintain high sperm production of good quality during more than 3 consecutive years. Under such conditions, the number of deep-frozen sperm doses is much higher than that of control bucks (from 41 to $69 \%$ more). Fertility of artificially inseminated goats is not different from that of goats inseminated with semen of control bucks.

Melatonin implants Melovine ${ }^{\circledR}$ had obtained in 1995, the official registration in France.

CHEMINEAU P., MALPAUX B., PELLETIER J., LEBOEUF B., DELGADILLO J.A., DELETANG F., POBEL T., BRICE G., 1996. Emploi des implants de mélatonine et des traitements photopériodiques pour maîtriser la reproduction saisonnière chez les ovins et les caprins. INRA Prod. Anim., 9 (1), 45-60. 